Case Report

\title{
A New Side Effect of Intravitreal Dexamethasone Implant (Ozurdex $\left.{ }^{\circledR}\right)$
}

\author{
Erdem Eris, Gurkan Erdogan, Irfan Perente, Gokhan Demir, \\ Burcin Kepez Yildiz, and Ebru Demet Aygit
}

Dr. Resat Belger Beyoglu Eye Training and Research Hospital, Istanbul, Turkey

Correspondence should be addressed to Erdem Eris; erdem-eris@hotmail.com

Received 22 June 2017; Revised 6 August 2017; Accepted 27 August 2017; Published 2 October 2017

Academic Editor: Maurizio Battaglia Parodi

Copyright (C) 2017 Erdem Eris et al. This is an open access article distributed under the Creative Commons Attribution License, which permits unrestricted use, distribution, and reproduction in any medium, provided the original work is properly cited.

\begin{abstract}
Dexamethasone implant, 0,7 mg (Ozurdex, Allergan, Inc., Irvine, CA, USA), is drug mostly used in the treatment of the diabetic macular edema and edema related to retinal vein occlusion. By reporting this case we aimed to report a new side effect of $0.7 \mathrm{mg}$ intravitreal dexamethasone implant that has not been reported in the literature before.
\end{abstract}

\section{Introduction}

Central serous chorioretinopathy (CSC) is a disorder characterized by serous retinal detachment and/or retinal pigment epithelial (RPE) detachment, changes that are most often confined to the macula and due to leakage of fluid through the RPE into the subretinal space $[1,2]$. CSC is a relatively rare cause of visual impairment, which typically affects the adult males (20-50 years) [3]. No case has been reported in individuals younger than twenty before [4]. CSC has been associated with the use of agents such as endogenous hypercortisolism and epinephrine. Iatrogenically systemic corticosteroids have been charged as a cause of CSC [5]. But CSC after local intraocular dexamethasone implantation has not ever been reported yet. The overactivation of mineralocorticoid receptor pathway in choroid vessels is still unknown etiology for CSC [6]. Hypertension, pregnancy, dialysis, organ transplantation, and systemic lupus erythematosus are entities that could result in choroidal vascular dysfunction and CSC $[5,6]$.

To the best of our knowledge, this is the first case report in the literature where CSC occurred following intraocular dexamethasone implantation.

\section{Case Report}

A 46-year-old male patient with a history of diabetes mellitus applied to our clinic with best corrected visual acuity (BCVA) of $80 / 200$ for the right eye (OD) and 40/200 for the left one (OS) (Figure 1). The patient had applied to both eyes the $0.7 \mathrm{mg}$ dexamethasone intravitreal implant (Ozurdex) for the treatment of diabetic macular edema. After a month of these applications, the best corrected visual acuities were 40/200 OD and 10/200 OS. Fundus examination revealed a macular serous detachment of both eyes. Both eyes had a large serous neurosensory detachment on optical coherence tomography (OCT) (Heidelberg Engineering, Heidelberg, Germany) (Figure 2). Although typical CSC findings (round, welldelineated serous detachment, massive choroid thickness, and pigment epithelial detachment [PED]) were not seen on OCT, other differential diagnoses of CSC were eliminated by fundus fluorescein angiography (FFA), Indocyanine Green Angiography (ICGA), and fundus examination. On ICGA there was only choroidal vascular enlargement (Figure 3). Upon slit lamp examination, there was no evidence of inflammation in the anterior or posterior chamber. There were no notable findings that can cause CSC performed in the Endocrine Department. The patient was diagnosed with dexamethasone intravitreal implant induced CSC, whose clinical course was monitored without any specific treatments. Two months following a monitoring regimen, fundoscopy and OCT were performed (Heidelberg Engineering, Heidelberg, Germany). Serous retinal detachments levels had decreased and the best corrected visual acuity in the both eyes had increased (Figure 4). Three months later both eye's serous 


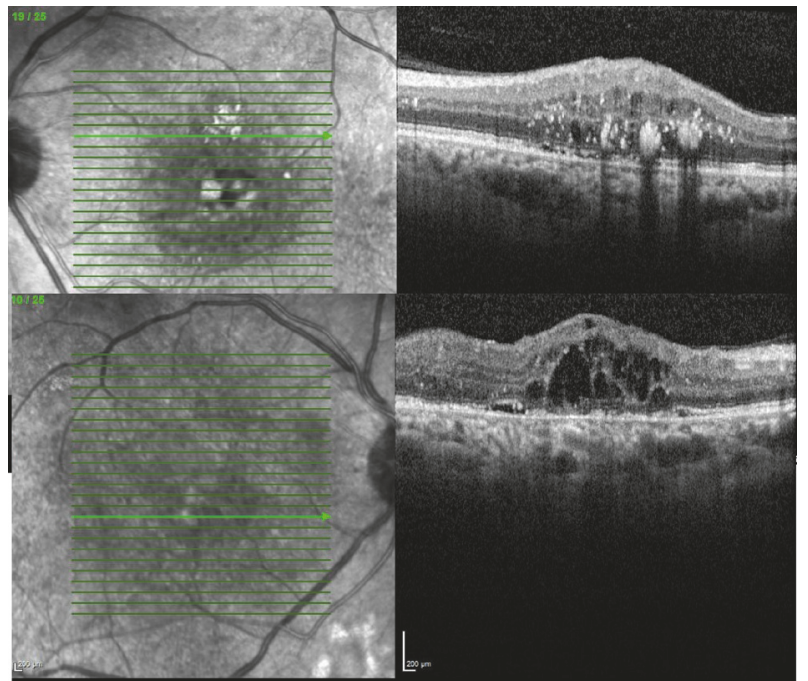

FIGURE 1: Macular edema secondary to diabetic retinopathy was seen on left eye's OCT image (superior image). Macular edema secondary to diabetic retinopathy was seen on right eye's OCT image (inferior image).

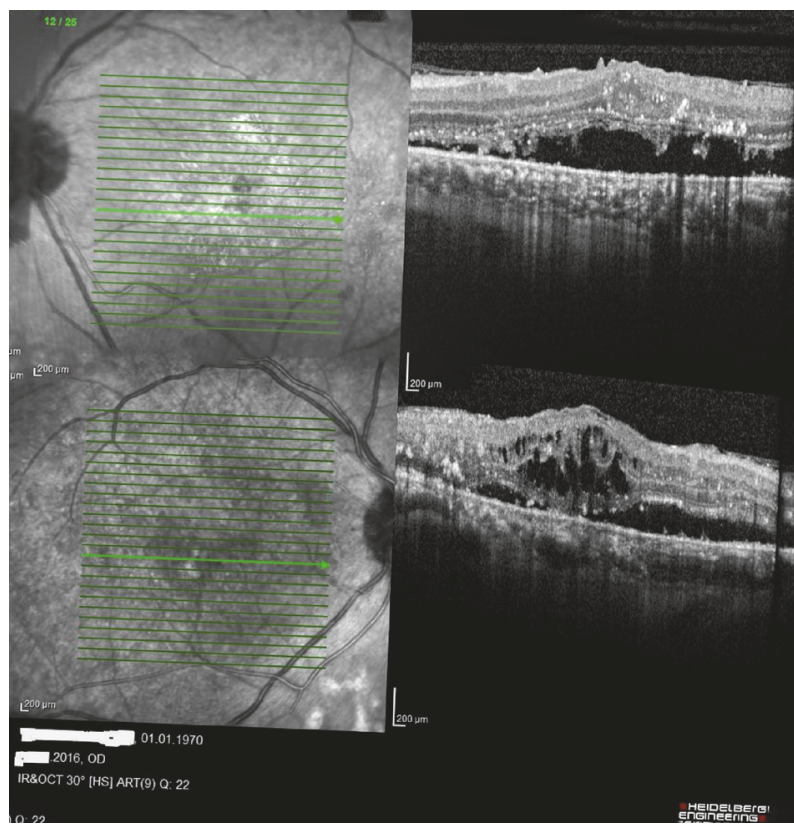

FIGURE 2: Central serous chorioretinopathy was seen on left eye's OCT image (superior image). Central serous chorioretinopathy was seen on right eye's OCT image (inferior image).

retinal detachments had disappeared (Figure 5). Final BCVA were 100/200 OD and 50/200 OS.

\section{Discussion}

Central serous chorioretinopathy is a chorioretinal disease whose pathogenesis remains obscure; however recent studies show its vascular origin. The reduced choroidal perfusion

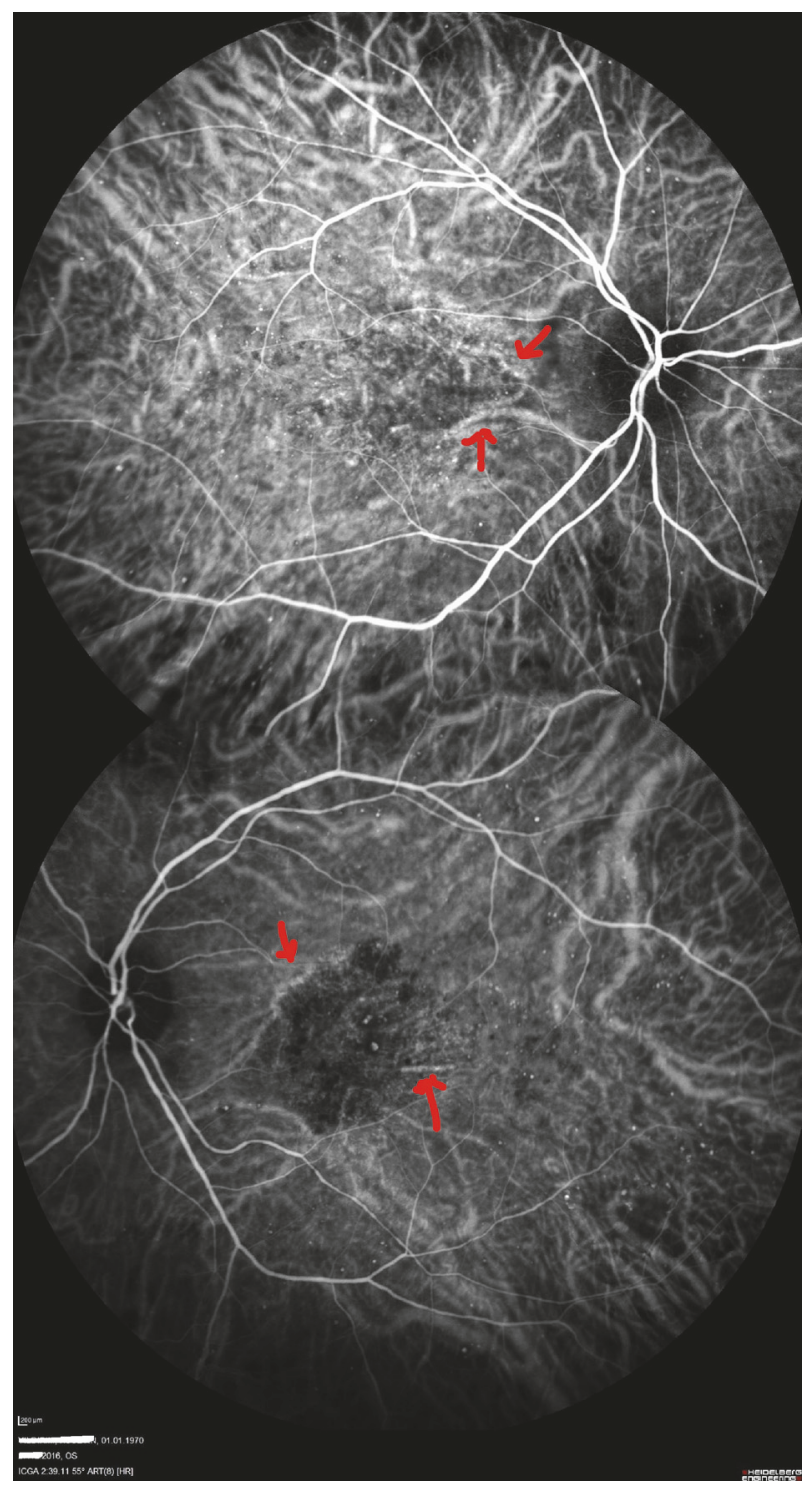

FIGURE 3: Indocyanine Green Angiography images (superior image is right eye and inferior image is left eye). Red arrows show choroidal vascular enlargement.

is believed to occur secondary to vasomotor instability or sympathetic nervous system excitation $[4,5]$.

CSC mostly occurs between 20 and 45 years of age. That is because if patients are over 50 years then macular degeneration, choroidal neovascularization, or polypoidal choroid vasculopathy should be ruled out for CSC diagnosis [7]. OCT and ICG may help to differentiate from other diagnoses [8]. In our case, other differential diagnoses of CSC were eliminated with fundus fluorescein angiography (FFA), Indocyanine Green Angiography (ICGA), and fundus examination.

But approximately $50 \%$ of patients may develop recurrence [9] and a chronic disease that can produce decreased visual acuity. Chronic CSC could be treated by the effectiveness of laser therapy and photodynamic therapy (PDT). Although systemic and topical steroid-induced central serous 


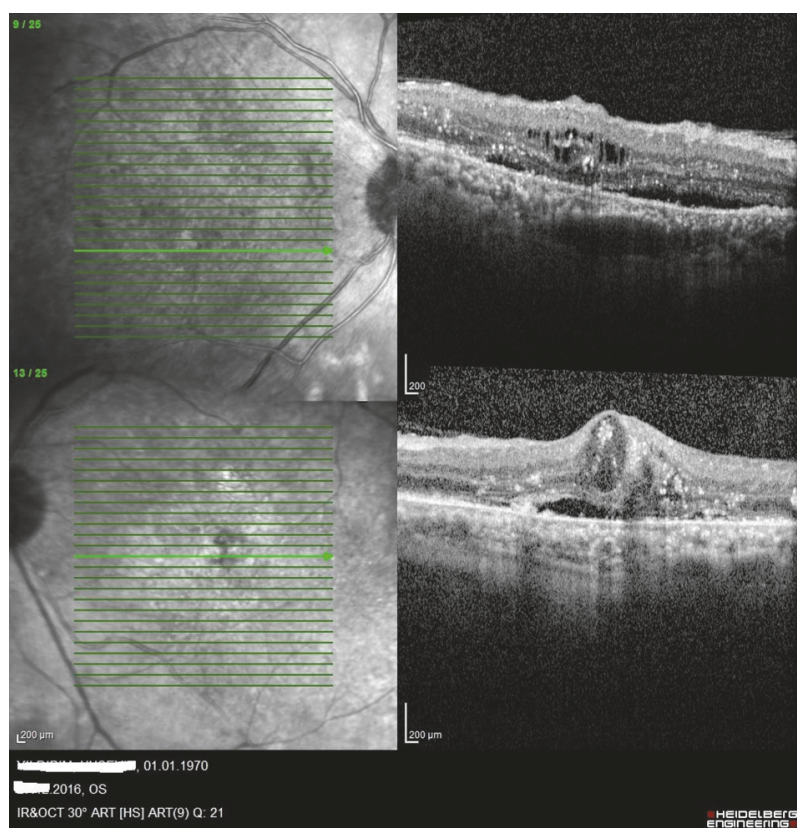

FIGURE 4: Serous retinal detachments levels were decreased (superior image is right eye and inferior image is left eye).

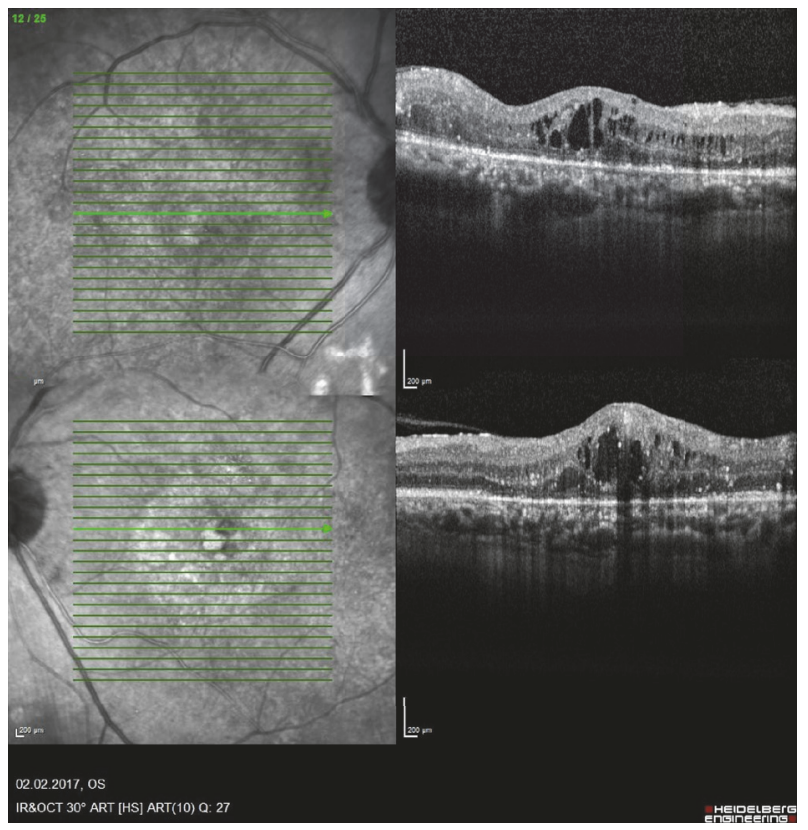

FIGURE 5: Three months later both eye's serous retinal detachments disappeared (superior image is right eye and inferior image is left eye).

chorioretinopathy was reported previously, dexamethasone intravitreal implant induced CSC was not reported before [10]. On the other hand two months later although dexamethasone intravitreal implant effect is expected to continue, we observed a decrease on the serous detachment of the CSC. It would be relevant to the peak time of dexamethasone release. But more studies are necessary to cast light on its effect.

\section{Ethical Approval}

All works were conducted in accordance with the Declaration of Helsinki (1964).

\section{Consent}

Consent for publication of figures and ophthalmic images was obtained from the patients.

\section{Conflicts of Interest}

The authors declare that they have no conflicts of interest. None of the authors has financial or proprietary interests in any material or method mentioned.

\section{References}

[1] C. Prünte and J. Flammer, "Choroidal Capillary and Venous Congestion in Central Serous Chorioretinopathy," Am J Ophthalmol, vol. 121, pp. 26-34, 1996.

[2] M. Wang, I. C. Munch, P. W. Hasler, C. Prünte, and M. Larsen, "Central serous chorioretinopathy," Acta Ophthalmologica, vol. 86, no. 2, pp. 126-145, 2008.

[3] R. Levine, A. J. Brucker, and F. Robinson, "Long-term followup of idiopathic central serous chorioretinopathy by fluorescein angiography," Ophthalmology, vol. 96, no. 6, pp. 854-859, 1989.

[4] S. A. B. Sibayan, K. Kobuch, D. Spiegel et al., "Epinephrine, but not dexamethasone, induces apoptosis in retinal pigment epithelium cells in vitro: Possible implications on the pathogenesis of central serous chorioretinopathy," Graefe's Archive for Clinical and Experimental Ophthalmology, vol. 238, no. 6, pp. 515-519, 2000.

[5] A. V. Chappelow and M. F. Marmor, "Multifocal electroretinogram abnormalities persist following resolution of central serous chorioretinopathy," Archives of Ophthalmology, vol. 118, no. 9, pp. 1211-1215, 2000.

[6] L. Y. Chan, R. S. Adam, and D. N. Adam, "Localized topical steroid use and central serous retinopathy," Journal of Dermatological Treatment, vol. 27, no. 5, pp. 425-426, 2016.

[7] W. M. Hart Jr., Adler's Physiology of the Eye, St. Louis: Mosby Year Book, 9th edition, 1992.

[8] T. Maaranen and M. Mäntyjärvi, "Contrast sensitivity in patients recovered from central serous chorioretinopathy," International Ophthalmology, vol. 23, no. 1, pp. 31-35, 1999.

[9] H. M. Leibowitz, D. E. Krueger, L. R. Maunder, R. C. Milton, M. M. Kini, H. A. Kahn et al., "The Framingham Eye Study monograph: an oph-thalmological and epidemiological study of cataract, glaucoma, diabetic retinopathy, macular degeneration, and visual acuityin a general population of 2631 adults, 1973-1975," Surv Oph-Thalmol, vol. 24, pp. 335-610, 1980.

[10] Y. C. Kim, S. D. Kim, and K. S. Kim, "A case of idiopathic central serous chorioretinopathy in a 12 -year-old male treated with bevacizumab., Korean journal of ophthalmology : KJO, vol. 26, no. 5, pp. 391-393, 2012. 


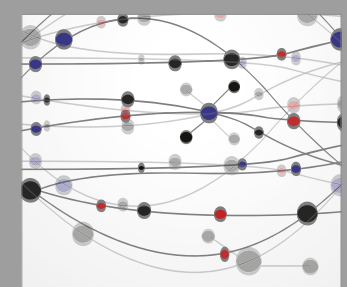

The Scientific World Journal
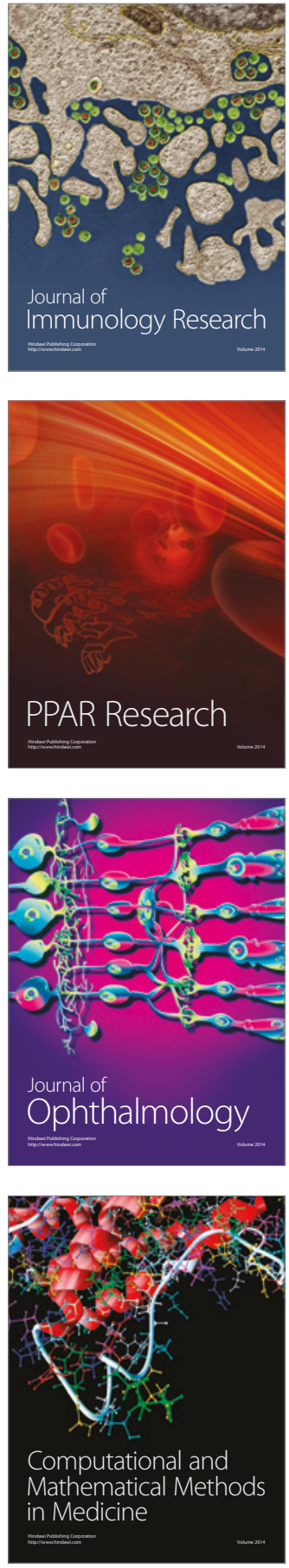

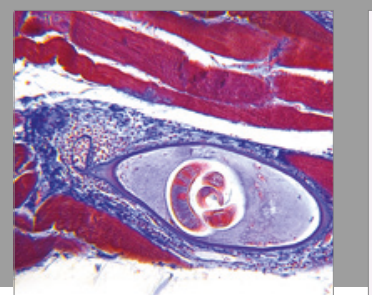

Gastroenterology Research and Practice
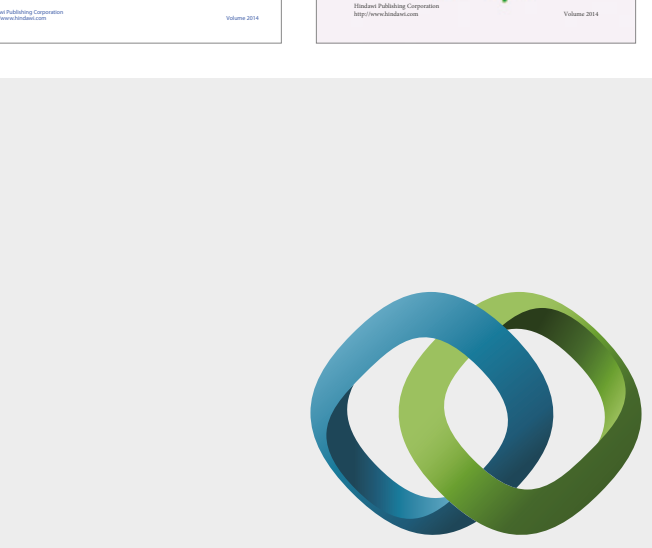

\section{Hindawi}

Submit your manuscripts at

https://www.hindawi.com
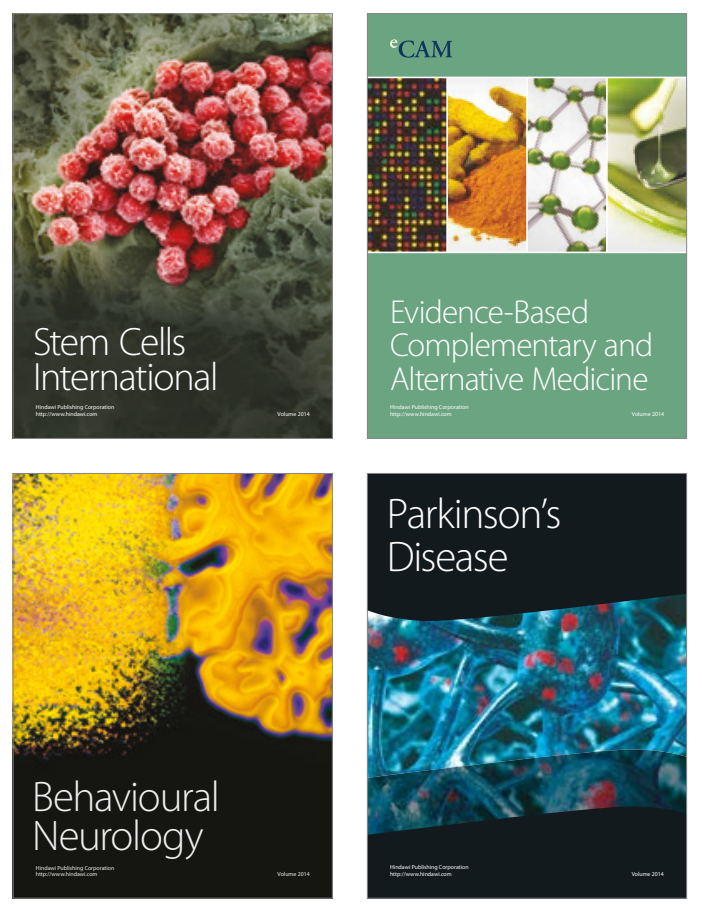
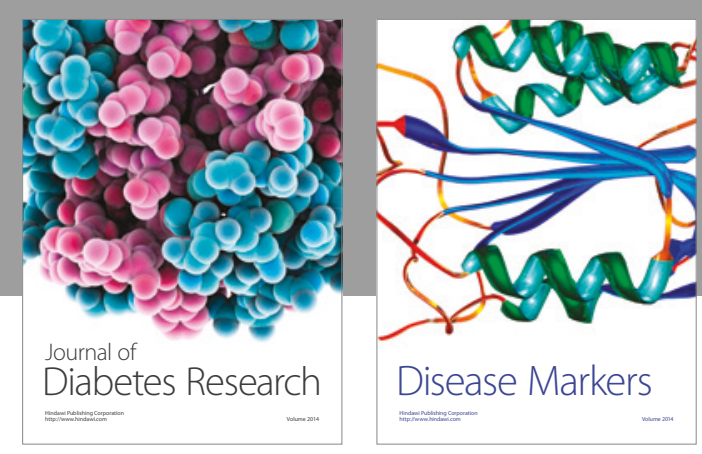

Disease Markers
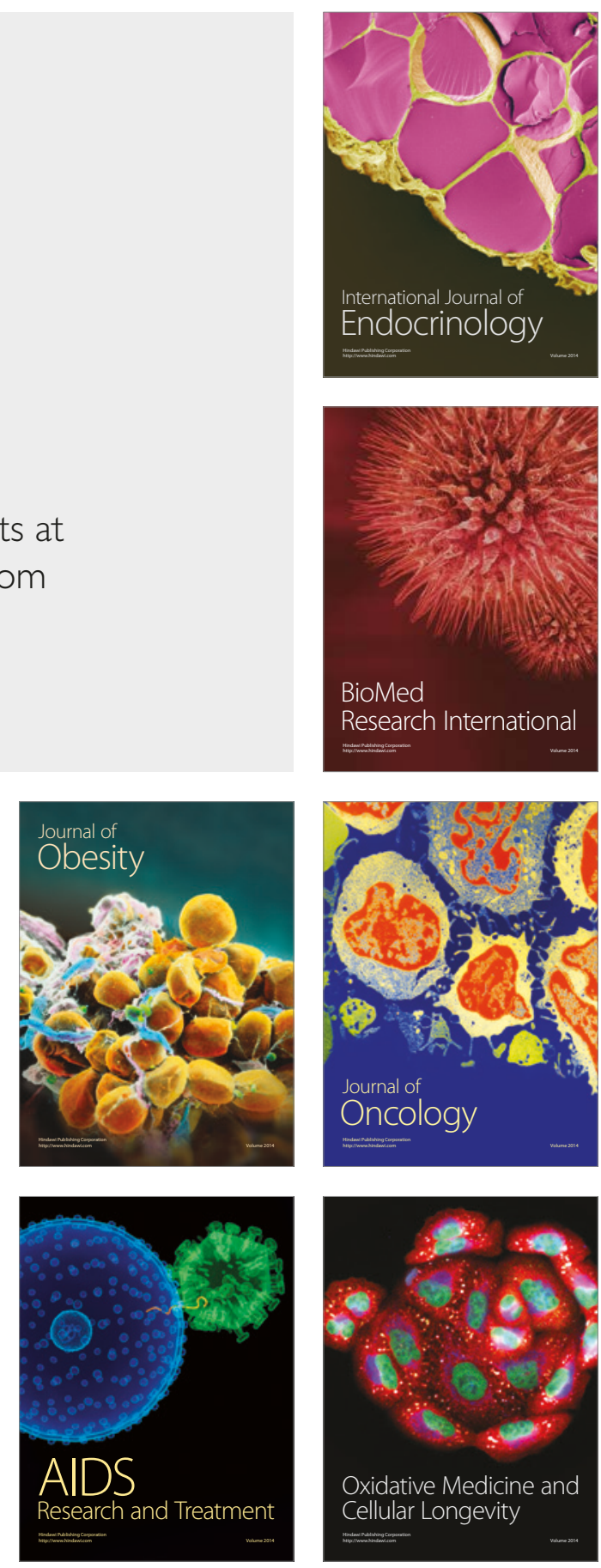